\title{
Water vapor in the atmosphere: an examination for CARMA phase correction
}

Yu-Shao Shiao, Leslie W. Looney, David P. Woody, Richard L. Plambeck, Alberto D. Bolatto

Yu-Shao Shiao, Leslie W. Looney, David P. Woody, Richard L. Plambeck, Alberto D. Bolatto, "Water vapor in the atmosphere: an examination for CARMA phase correction," Proc. SPIE 6275, Millimeter and Submillimeter Detectors and Instrumentation for Astronomy III, 62750Y (27 June 2006); doi: $10.1117 / 12.672245$

Event: SPIE Astronomical Telescopes + Instrumentation, 2006, Orlando, Florida , United States 


\title{
Water Vapor in the Atmosphere: An Examination for CARMA Phase Correction
}

\author{
Yu-Shao Shiao ${ }^{a}$, Leslie W. Looney ${ }^{a}$, David P. Woody ${ }^{b}$, Richard L. Plambeck ${ }^{c}$ and Alberto D. \\ Bolatto $^{c}$ \\ ${ }^{a}$ Univ. of Illinois at Urbana-Champaign, IL 61801, USA; \\ ${ }^{b}$ California Institute of Technology, CA 91125, USA; \\ ${ }^{c}$ Univ. of California, Berkeley, CA 94720, USA
}

\begin{abstract}
We report on the current progress of the water vapor radiometer (WVR) phase correction project for the Combined Array for Research in Millimeter-wave Astronomy (CARMA). CARMA is a new millimeter array that merges the Owens Valley Radio Observatory (OVRO) array, the Berkeley-Illinois-Maryland Association (BIMA) array and eventually the Sunyaev-Zel'dovich Array (SZA). WVRs are designed for phase correction by monitoring the water vapor in the atmosphere along the line of sight toward astronomical sources. In addition, we discuss the stability of the current OVRO water vapor radiometers in preparation for testing at the CARMA site. We will systematically analyze the receivers with atmospheric correlations to decouple the effects of instrumentation and atmospheric noise. Finally, we report on the status of the correlation receivers in development.
\end{abstract}

\section{INTRODUCTION}

In 2004, construction began on the new Combined Array for Research in Millimeter-wave Astronomy (CARMA) project $^{1}$ by moving the Berkeley-Illinois-Maryland Association (BIMA) and the Owens Valley Radio Observatory (OVRO) antennas to the CARMA site. The site, Cedar Flat in eastern California, is at 7200 feet elevation, which significantly reduces the effect of atmospheric decorrelation. The collecting area of CARMA is about $725 \mathrm{~m}^{2}$ with eventual long baselines of $2 \mathrm{~km}$. All merged, these attributes increase the observing efficiency by $50 \%-100 \%$, compared to the BIMA or OVRO arrays. CARMA is currently ongoing final integration and was dedicated on May 5th, 2006.

However, even with a better site, CARMA will still suffer from atmospheric fluctuations. For interferometers, atmospheric fluctuations decrease phase coherence and observation quality. Observing phase calibrators such as quasars, can only recover atmospheric phase variations on time scales of 1 hour. To correct phase variation on shorter time scales, a CARMA water vapor radiometer (WVR) system is being developed to measure and correct for the twinkling of phase shifts from fluctuating amounts of tropospheric water vapor to increase the sensitivity of the array. There are two water emission lines for remote sensing of the water vapor column in the microwave band: the $6_{16}-5_{23}$ rotational line transition near $22 \mathrm{GHz}$ and the $3_{13}-2_{20}$ rotational line transition near $183 \mathrm{GHz}$. Early experiments and modeling of the water vapor emission by the consortium determined that the optically thin $22 \mathrm{GHz}$ line was the best phase correction method for the OVRO and BIMA sites. The $183 \mathrm{GHz}$ emission line, although very sensitive to small water columns, starts to become optically thick with moderate water vapor (about $2 \mathrm{~mm}$ of precipitable water vapor, or PWV). The BIMA/OVRO sites routinely observed with 8 to $9 \mathrm{~mm}$ of PWV.

The opacity of Cedar Flat was measured by the atmospheric total power using a $225 \mathrm{GHz}$ tipper during three periods: 2002 October - 2003 January, 2003 February - 2003 May, and 2003 August - February 2004. Figure 1 illustrates the opacity as a percent of the observing time during these periods (i.e. $50 \%$ of the time the opacity was below 0.26 ). Table 1 summarizes the measured opacity and uses a simple atmospheric model to calculate the associated PWV. The opacity data implies that $50 \%$ of the time the PWV is less than $5 \mathrm{~mm}$, but only $5 \%$ of the time is it less than $2 \mathrm{~mm}$, suggesting again that the optically thin line at $22 \mathrm{GHz}$ is the preferred remote sensing approach for CARMA.

Author information: E-mail: shaw2@astro.uiuc.edu

Millimeter and Submillimeter Detectors and Instrumentation for Astronomy III, edited by Jonas Zmuidzinas, Wayne S. Holland, Stafford Withington, William D. Duncan Proc. of SPIE Vol. 6275, 62750Y, (2006) · 0277-786X/06/\$15 - doi: 10.1117/12.672245

Proc. of SPIE Vol. $627562750 Y-1$ 


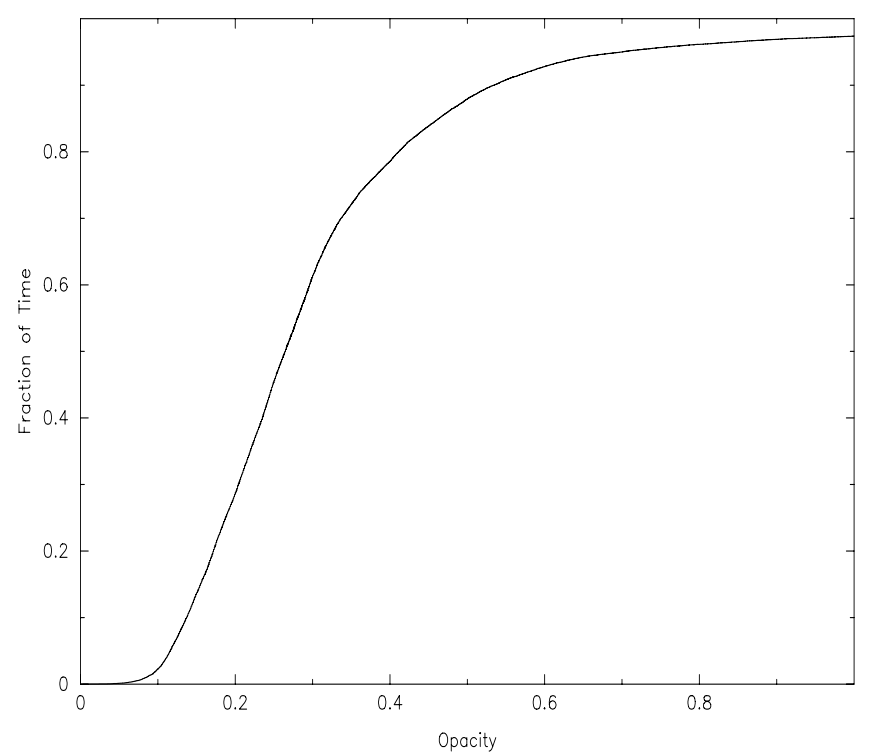

Figure 1. Results from a $225 \mathrm{GHz}$ tipper located at the Cedar Flat CARMA site. The horizontal axis is the opacity measured by the tipper and the vertical axis is the fraction of the time that the opacity was that value or lower. The data was collected from 2002 October - 2003 January, 2003 February - 2003 May, and 2003 August - February 2004.

\begin{tabular}{ccc}
\hline Percent of Time & Estimated Opacity & Estimated PWV (mm) \\
\hline $90 \%$ & 0.53 & 12 \\
$50 \%$ & 0.26 & 5 \\
$25 \%$ & 0.2 & 4.5 \\
$10 \%$ & 0.14 & 3 \\
$5 \%$ & 0.12 & 2 \\
\hline
\end{tabular}

Table 1. Cedar Flat Atmospheric Opacity 


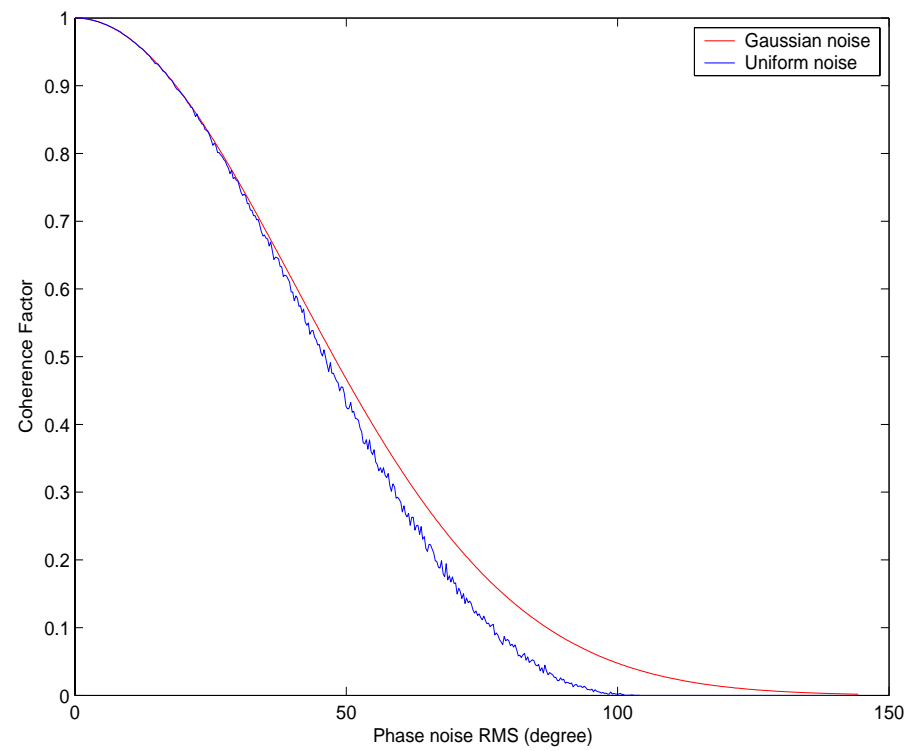

Figure 2. The coherence factor versus phase noise RMS. The $\mathrm{x}$-axis is the phase RMS and the y-axis is the coherence factor. The coherence factor is defined as the ratio of the receiving signal strength to the maximum signal strength.

In addition to the CARMA effort, as well as the former $\mathrm{OVRO}^{2}$ and $\mathrm{BIMA}^{3}$ efforts, there are numerous ongoing projects to develop reliable phase correction WVRs, using either $22 \mathrm{GHz}$ or $183 \mathrm{GHz}$ radiometers, for many radio telescope interferometers including the Very Large Array (VLA), ${ }^{4}$ the Institute for Radio Astronomy at Millimeter wavelengths (IRAM) ${ }^{5}$ the Submillimeter Array (SMA) ${ }^{6}$ and the eventual Atacama Large Millimeter Array (ALMA). ${ }^{7}$

\section{WATER VAPOR PHASE CORRECTION}

The CARMA consortium has an extensive history investigating the atmospheric fluctuations due to variations in the water vapor column above each antenna and their subsequential correction. The decorrelation from this radio "seeing" limits the overall sensitivity and dynamic range of the interferometric maps, more so with short wavelength or long baseline observations.

\subsection{Phase Coherence versus Signal Strength}

To demonstrate the importance of the phase decorrelation in interferometry, we assume two models for the dechoerence of the atmosphere. One is Gaussian distributed phase noise, which can be solved analytically. ${ }^{8}$ The other is uniformly distributed phase noise, which is calculated numerically. We explore the phase noise effect and compare spectra with WVR phase correction.

Figure 2 shows the coherence factor versus the phase noise RMS, where the coherence factor is defined as the receiving power ratio of partially coherent waves and perfectly coherent waves for both cases. We assume that atmospheric absorption is not involved, i.e. only the phase noise affects the signal strength. For both cases, when the phase noise RMS is $0^{\circ}$, the waves are perfectly coherent and the coherence factor is 1 . For the uniform distributed phase noise, when the RMS is $103.9^{\circ}$, the waves are completely incoherent and the coherent factor is 0 . For a Gaussian distributed phase noise the coherence factor will be slightly higher than the former. When the atmosphere is stable, phase noise on short time scales tends to satisfy the Gaussian distribution. Given system phase noise, one can quantitatively determine the data quality.

To explore the impact of the atmospheric phase RMS on observations, we used the MIRIAD ${ }^{9}$ task uvgen that includes phase RMS variation in simulated observations, assuming uniform distributed phase noise. In Figure $3(\mathrm{a})$, we show a simulated observation with an overall system noise of about $0.16 \mathrm{Jy}$ and a source with a spectral 

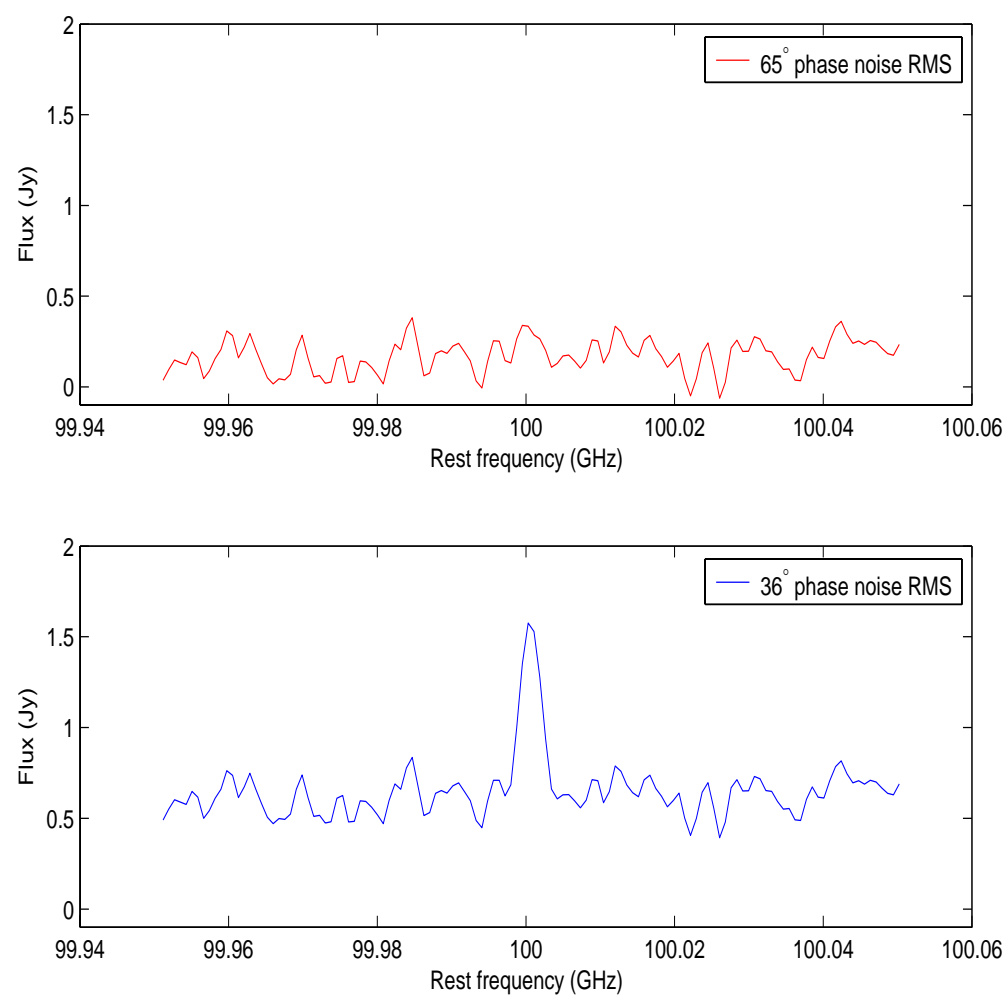

Figure 3. (a) The plot on the top shows a $100 \mathrm{GHz}$ spectral line with $65^{\circ} \mathrm{RMS}$ of the phase noise. It is not detected due to low Signal to Noise ratio $(\mathrm{S} / \mathrm{N})$. (b) The plot on the bottom shows the spectrum improved by lowering the RMS to $36^{\circ}$ with the WVR phase correction, a $3 \sigma$ detection.

line $2 \mathrm{Jy}$ above a $1 \mathrm{Jy}$ continuum, where these parameters are chosen for convenience. In the case of $65^{\circ}$ phase noise RMS, the strength of both the spectral line and the continuum drop below the noise power, making this a non-detection, even as the spectral line is theoretically detectable. Assuming that the WVR system can lower the phase noise RMS to $36^{\circ}$, in Fig. $3(\mathrm{~b})$, the detection of the spectral line is clearly improved to $>3 \sigma$. Clearly, phase coherence plays a critical role in weak line detection observations.

\subsection{Delay Path and Water Vapor Relationship}

Water vapor is a major component altering the refraction index of the atmosphere and distorting microwave fronts of astronomical sources. ${ }^{10}$ Empirically, the pathlength variation $L_{w}$ of the atmosphere can be obtained from $(1)^{8}$

$$
L_{w}\left(\rho_{w}, T\right)=0.299 \times P W V+1742.2 \times 10^{-6} \int_{\text {ground }}^{\infty} \frac{\rho_{w}(s)}{T(s)} d s
$$

where $T$ is the temperature function and $\rho_{w}$ is the water vapor density function. Both functions are in terms of the height, s. $P W V$ stands for the perceptive water vapor in millimeter $(\mathrm{mm})$. The second term on the right-hand side in Equation (1) is an integral from the ground to infinite height. As long as $\rho_{w}(s)$ and $T(s)$ are known, one can determine the path delays of each antenna and apply phase correction by comparing the differences of path delays. Water vapor radiometers (WVRs) are designed to detect the atmospheric water vapor above an antenna that relates to the phase fluctuations. For the optically thin $22 \mathrm{GHz}$ water vapor line, the translation from the intensity of the spectral line to the path delay should be a simple linear scale factor. 


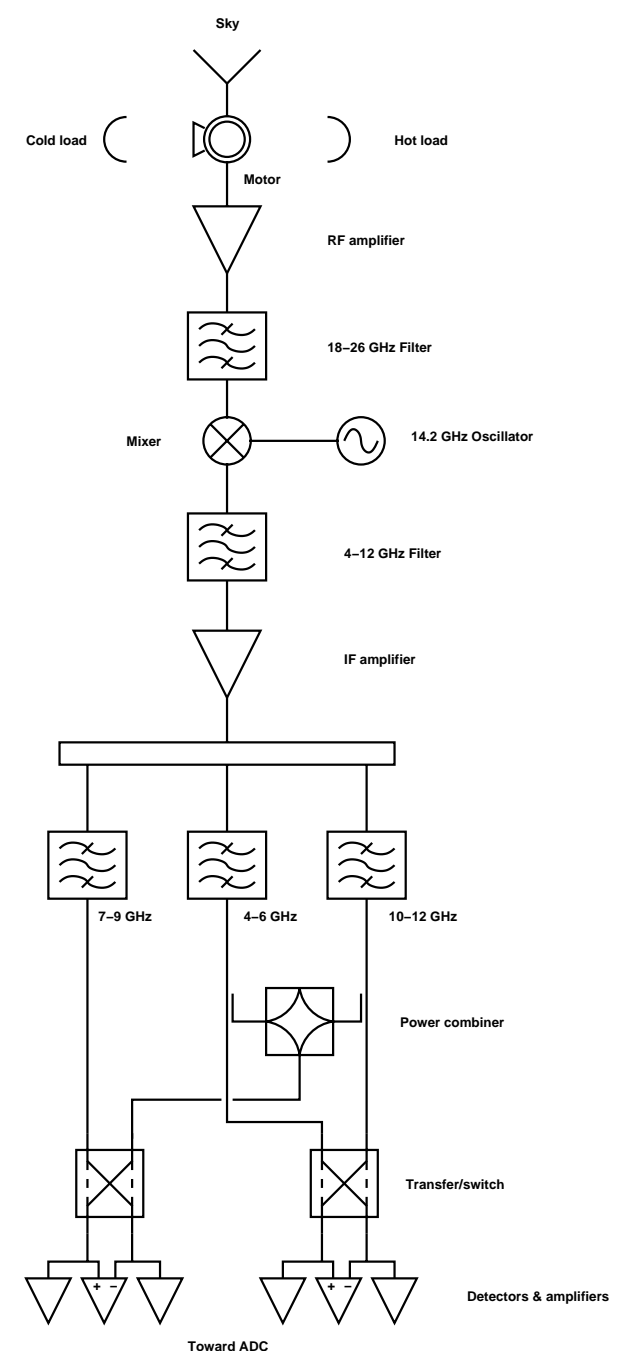

Figure 4. The system block diagram of the OVRO WVRs.

\section{WATER VAPOR RADIOMETER HISTORY IN THE CONSORTIUM}

Millimeter wave continuum radiometry at BIMA using total power receivers ${ }^{3}$ (requiring a stability of $10^{-4}$ ) was successful only under certain atmospheric conditions. Analysis of the total power data showed that only in clear weather was there any correlation between the atmospheric phase fluctuations and the total power measurements. ${ }^{11}$ During those times, the phase correction was successful only about $50 \%$ of the time.

At OVRO, WVRs were installed in $1999^{2}$ with cryogenic cooled $(15 \mathrm{~K})$, low-noise $18-26 \mathrm{GHz}$, InP amplifiers Fig. 4. These receivers were an upgrade to previous less sensitive uncooled systems. Basically, the OVRO WVRs are total-power systems with double side-band receivers, which is a simple and robust design. As shown in Figure 4, the receiver includes radio frequency (RF) amplifiers, band pass filters, power combiners/splitters, and detectors. The water vapor emission power goes into the horn antenna and is amplified by the RF amplifier. The horn is driven by a motor so it can observe sources from different directions: sky, hot load, or cold load. The band pass filter behind the RF amplifier is designed with 18-26 GHz pass band so the water vapor signal can go through the mixer without interference from other frequency signals. The mixer shifts the signal to a lower frequency band. An intermediate frequency (IF) filter and IF amplifiers can increase the magnitude of the signal again. 
There are three output bands in the power splitter, 4 to $6 \mathrm{GHz}, 7$ to $9 \mathrm{GHz}$ and 10 to $12 \mathrm{GHz}$. They are filtered separately by band-pass filters and later combined by power combiners. To decrease mis-match effects between different channels, two switches are installed. Finally, the signals are amplified and converted to low frequency signals by diode detectors. After the signals come out of the WVR, the data are acquisitioned. The sensitivity needed for detecting water vapor suffers from fluctuations in system gain, offset, bandwidth and noise temperature. To stabilize the WVRs, constant calibration of these parameters is done by observing artificial sources, the hot load and the cold load.

By analyzing the last few years of data, the residual delay errors of 100-200 $\mu \mathrm{m}$ are obtained under some conditions by cross-calibrating the temperature scales of the WVRs and linking the delay phase of the target source to that of the a phase calibrator at a different elevation. Point source observations show that high quality images can be extracted from previously unusable data when the corrections are applied.

However, the general result is that the WVRs increase phase coherence less than $50 \%$ of the time. Although the accurate measurement of the relative gains and offsets of the $\mathrm{InP}$ amplifiers is the most critical and difficult step in the this phase correction approach, the simplified conversion factor may play a large role as well. The results gathered to date suggest that using a simple constant conversion factor to infer the phase correction from the $22.2 \mathrm{GHz}$ line intensity does not work consistently and that there may be further atmospheric physics at play. There is a trend that the worse the initial phase coherence the more likely the phase coherence will be improved by the assumption of a constant atmospheric conversion factor. The problem is that during median weather, when the phase correction should also be improving the observations, the water vapor emission does not well track the phase fluctuations. So although often the coherence is increased by tracking the atmospheric water vapor, we need to increase the robustness of this system.

At BIMA (April 1999), similar $22 \mathrm{GHz}$ water line radiometry experiments were performed on three BIMA antennas ${ }^{12}$ with two types of backends: a one channel swept frequency system and a 16 channel $4 \mathrm{GHz}$ bandwidth autocorrelator spectrometer (the Atmospheric Phase Inference Device, or APHID ${ }^{13}$ ). These tests also showed that about $50 \%$ of the time the interferometry data were improved. Unfortunately in the other $50 \%$ of the data, drifts in the measurement degraded the RMS phase. Again, an atmosphere scale factor was used instead of an atmospheric model. From this work, it was quickly realized that one of the limiting factors was the gain fluctuation in the system that was degrading the data.

\section{CURRENT PROGRESS}

Decorrelation caused by atmosphere-induced phase fluctuations across the array has effects similar to optical "seeing", limiting sensitivity, dynamic range, and angular resolution attainable by interferometric arrays. Our initial results at correcting these atmospheric phase fluctuations using WVRs to improve the phase coherence are very encouraging. By using the simplest assumptions about the atmospheric properties (i.e. a single conversion factor from water vapor to delay), WVR corrections improved the phase coherence more than $50 \%$ of the time. Moreover, most of the data in the worst atmospheric conditions improved by up to $60 \%$ with this simple approach.

Nonetheless, further research into phase correction is clearly needed if this technique is to evolve into a robust phase correction scheme for CARMA. In our experiments the phase residuals after application of the WVR corrections are larger than the expected noise from the WVR receivers, implying that our atmospheric "model" is too simplistic, and/or the receivers have low level instabilities hampering the WVR corrections. The CARMA consortium goals for a robust phase correction research program is to further investigate the importance of these two factors: ongoing development of stable correlation receivers and re-investigating more sophisticated treatment of the atmosphere properties with improved OVRO systems.

\subsection{Correlation Receivers}

One of the long-term problems in this field has been the question of receiver stability: are the observed fluctuations the instrument or the atmosphere? The development of an ultra-stable correlation receiver at $22 \mathrm{GHz}$ will provide the most stable system for WVR phase correction experiments. As shown in Figure 5, the system includes a $180^{\circ}$ hybrid, RF amplifiers, mixers and low pass filters for two channels. ${ }^{10}$ The gain calibration and signal detection are processed simultaneously. One input branch of the hybrid contains the sky signal from the horn and antenna 


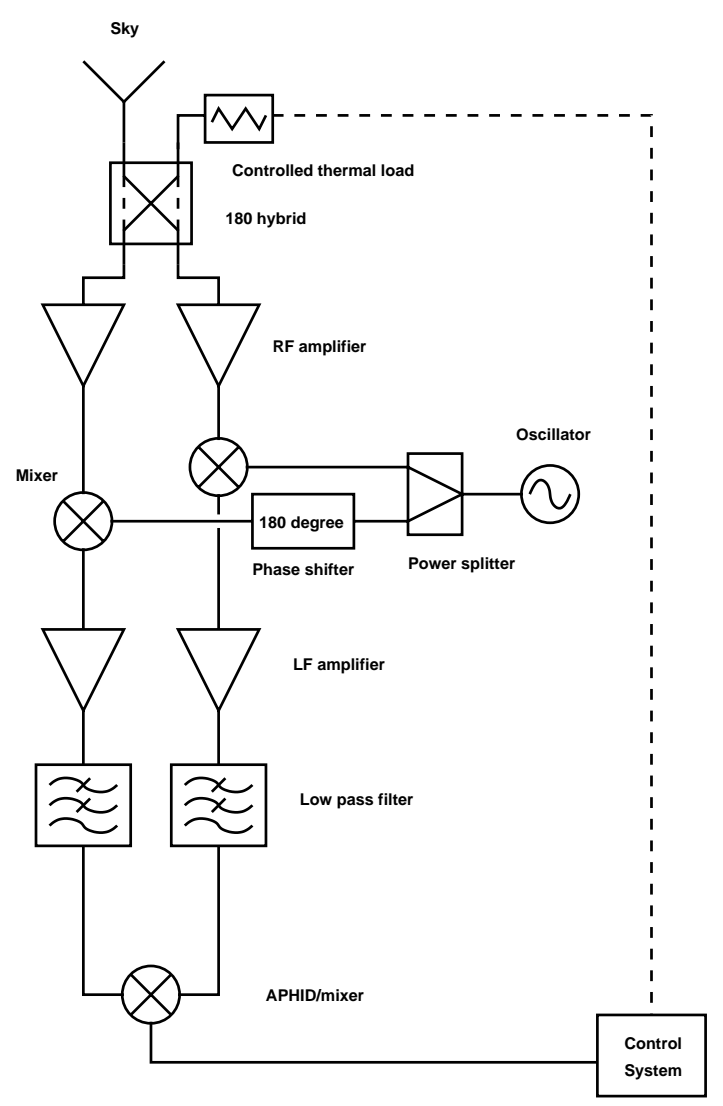

Figure 5. The system block diagram of the BIMA WVRs.

optics. The second input branch of the hybrid is connected to a calibration load. The calibration load has a variable temperature, constituting a controlled thermal load (CTL). Its functionality is similar to a noise source in a noise-adding receiver system, allowing the calibration of the system gains. The two output branches of the hybrid, after amplification and down-conversion, are multiplied together by a low frequency mixer and read-out by the control system. Because the calibration and sky signals propagate simultaneously through the same paths, the gain of either output branch is a multiplicative factor to the difference signal reconstituted by the low frequency multiplier. To measure the sky power, the CTL temperature is controlled to keep the power difference signal as small as possible, at which point the CTL temperature and the sky temperature are the same and the control system obtains the sky power measurement.

A lab prototype scanning system was assembled at Berkeley in 2001 by two of the authors (Bolatto and Plambeck), and successfully measured the $22 \mathrm{GHz}$ water vapor line spectrum. The production prototype, including a dewar, cryogenic amplifiers and CTL, a microprocessor controller, and a signal correlator was developed by 2004, but further development has been stalled in favor of higher priority CARMA integration tasks. The fundamental challenge of correlation radiometers is to minimize input mismatch at or before the first amplification stage, which is connected to the hybrid, and the mismatch results in spurious correlation power that could be interpreted as a temperature difference between the sky and the load. The difference between CTL physical and radiometric temperatures are also crucial to WVR data. Finally, CTL temperature agility is extremely important when the WVR tracks the varying sky temperature on short time scales. Therefore, Bolatto and Plambeck developed a low heat capacity, electronically controlled $50 \Omega$ microstrip load, which is capable of changing its physical temperature by tens of Kelvin in a few hundred milliseconds. 


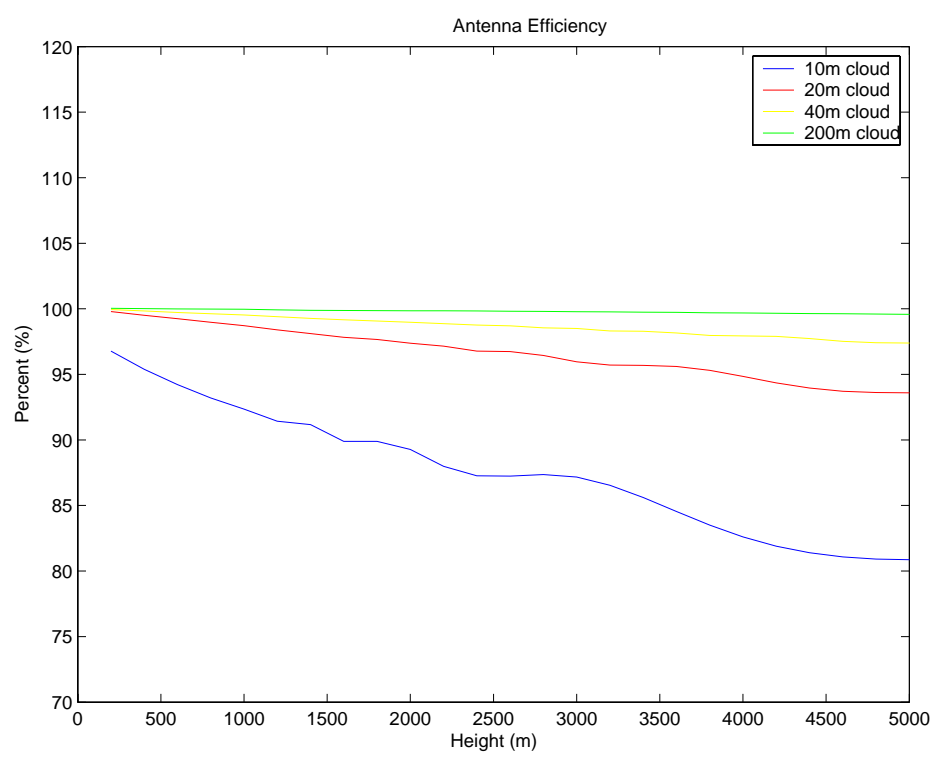

Figure 6. This plot shows the antenna efficiency with various finite-sized water vapor clouds at different heights above a $10-\mathrm{m}$ antenna. The antenna efficiency is the ratio of the antenna temperature to the water vapor temperature. The antenna efficiency of an infinite water vapor cloud is $100 \%$.

\subsection{Atmosphere and Instrument Stability}

Based on WVR observations from previous work, such as VLA ${ }^{4}$ and OVRO ${ }^{2}$ most of the time the scale factor of the $22 \mathrm{GHz}$ water vapor line is larger than expected from theory. Thus, we investigate this effect by numerically calculating antenna efficiency, which depends on the height in both near-field and far-field regions, to simulate water vapor detection. ${ }^{14}$ In the simulations, we define the antenna efficiency as the ratio of the receiving power from a finite-sized cloud to a infinite-sized cloud i.e. the ratio of the antenna temperature to water vapor cloud temperature. Water vapor radiometers are fundamentally single-dish total power receivers. We scan the antenna efficiency by putting a single layer of water vapor above a parabolic antenna and calculate the receiving power. By integrating the transmitting power from water vapor clouds to the antenna, one can compare with the infinitesized cloud and obtain the antenna efficiency. Figure 6 shows the antenna efficiency for a one-dimensional 10-m parabolic antenna with different-sized water vapor clouds at varying heights. For finite-sized water vapor clouds, the antenna efficiency decreases with height, which is similar to a beam filling factor, except the near and far fields effects are included in this simulation. Based on these simulations, we show that if the atmosphere fluctuations are small, the scale-factor will increase (in units of $\mu \mathrm{m} / \mathrm{mK}$ ). Clearly, one needs to better understand the atmospheric properties, including turbulence. The ALMA group has been working on the effects of turbulence in the atmosphere. ${ }^{15}$

During the earliest stages of CARMA, we will use the existing OVRO WVRs to systematically investigate both the instrument stability and the atmospheric properties at the CARMA site, e.g., what correlations with atmospheric conditions will emerge? The main goal over the next year is to employ the OVRO WVR systems and investigate the impact of atmospheric conditions on our ability to achieve phase correction at the Cedar Flat site. Analysis of the existing WVR data has been hampered by system reliability issues and the unavailability of atmospheric data on similar timescales: we will be tackling these two issues. The calibration and overall stability of the existing systems will be evaluated, characterized, and monitored. The 6 systems will be reinstalled on the CARMA 10-meter telescopes in late 2006. We will also emplace a system to gather atmospheric information (e.g., temperature, cloud cover, wind speed and direction, etc.) on timescales relevant to phase correction (seconds to minutes). With better characterized WVR systems and more comprehensive atmospheric data, we will address the main question suggested by the initial results described in Section 3: Are we missing any atmospheric physics? To answer this question, we will analyze the atmospheric data in conjunction with the 
water water vapor fluctuations and the interferometric phase variations.

\section{REFERENCES}

1. D. P. Woody, A. J. Beasley, A. D. Bolatto, J. E. Carlstrom, A. Harris, D. W. Hawkins, J. Lamb, L. Looney, L. G. Mundy, R. L. Plambeck, S. Scoot, and M. Wright, "Carma: a new heterogeneous millimeter-wave interferometer," in Advanced Technology MMW, Radio, and Terahertz Telescopes, J. Zmidzinas, W. S. Holland, and S. Withington, eds., Proc. SPIE 5498, pp. 30-41, 2004.

2. K. B. Marvel and D. P. Woody, "Phase correction at millimeter wavelengths using observations of water vapor at 22 ghz," in Advanced Technology MMW, Radio, and Terahertz Telescopes, T. G. Phillips, ed., Proc. SPIE 3357, pp. 442-452, 1998.

3. J. Staguhn, A. I. Harris, R. L. Plambeck, and W. J. Welch, "Phase correction for the bima array: atmospherical model caluclations for the design of a prototype correlation radiometer," in Advanced Technology MMW, Radio, and Terahertz Telescopes, T. G. Phillips, ed., Proc. SPIE 3357, pp. 432-441, 1998.

4. C. J. Chandler, W. F. Brisken, B. J. Bulter, and R. H. Hayward, "Results of water vapour radiometry tests at the vla," EVLA Memo 73, 2004.

5. M. Bremer, S. Guilloteau, and R. Lucas, "Atmospheric phase correction based on sky emission in the 210-248 ghz band," the ESO-IRAM-NFRA-Onsala Workshop on Science with Large Millimetre Arrays , 1995.

6. M. C. Wiedner, R. E. Hills, J. E. Carlstrom, and O. P. Lay, "Interferometric phase correction using 183 ghz water vapor monitors," ApJ 553, pp. 1036-1041, 2001.

7. R. Hills and J. Richer, "Water vapour radiometers for alma," ALMA Memo 303, 2000.

8. A. R. Thompson, J. M. Moran, and G. W. Swenson, Interferometry and Synthesis in Radio Astronomy, Addison-Wesley, Reading, Mass., 2001.

9. R. J. Sault, P. J. Teuben, and M. C. H. Wright, "A retrospective view of miriad," PASP Conf Series 77.

10. J. Waters, Methods of experimental physics: Astrophysics Part B: Radio Telescopes, Academic Press, Inc, New York, 1976.

11. M. C. H. Wright, "Atmospheric phase prediction from total power measurements," BIMA Memo 44, 1996.

12. M. C. H. Wright, "Atmospheric phase correction using water vapor radiometers," BIMA Memo 78, 2000.

13. A. Harris, "Precision radiometry and the aphid $22 \mathrm{ghz}$ water line monitor," BIMA Memo 80, 2000.

14. Y.-S. Shiao, L. W. Looney, and E. C. Sutton, "Exploring power patterns of parabolic antennas: Implications for water vapor radiometers," CARMA Memo in preparation, 2006.

15. A. Stirling, J. Richer, R. Hills, and A. Lock, "Turbulence simulations of dry and wet phase fluctuations at chajnantor. part i: The daytime convective boundary layer," ALMA Memo 517, 2005. 